\title{
The importance of Chakras and Energy Imbalances Correction in the Prevention and Treatment of Gestational Diabetes
}

\author{
Huang Wei Ling* \\ MD Infectious Diseases Doctor, General practice doctor, Nutrition doctor, Acupuncture doctor and Pain \\ management doctor, Medical Acupuncture and Pain Management Clinic, Franca, São Paulo, Brazil \\ *Corresponding Author: Huang Wei Ling, MD Infectious Diseases Doctor, General practice doctor, \\ Nutrition doctor, Acupuncture doctor and Pain management doctor, Medical Acupuncture and Pain \\ Management Clinic, Franca, São Paulo, Brazil, Email: weilingmg@gmail.com
}

\begin{abstract}
Statement of the Problem: Gestational Diabetes (GD) is high-blood sugar that develops during pregnancy and usually disappears after giving birth. In Traditional Chinese Medicine (TCM), the physiopathology of GD is Yin deficiency and Heat retention.
\end{abstract}

Purpose: To demonstrate the importance of chakras and energy imbalances corrections in the prevention and treatment of gestational diabetes.

Methodology: Three case reports. The first and second patients were diagnosed with GD in the second trimester of pregnancy, in the 21 and 28 weeks, respectively. The first patient was pregnant with twins, and the diagnoses happened after evaluation the babies' percentile (above 85) and glucose tolerance test that resulted $81 ; 231 ; 187 \mathrm{mg} / \mathrm{dl}$. In the second patient's case, her glucose tolerance test appeared $156 \mathrm{mg} / \mathrm{dl}$ (normal $140 \mathrm{mg} / \mathrm{dl}$ ). Both patients' chakras were at the level 1 of 8 , with significant lack of energy. The first patient had a previous chakras measurement, and was presenting energy deficiency a year before the pregnancy. The third patient had gestational diabetes in the last two of her three pregnancies, and after the third pregnancy, she was diagnosed with type 1 diabetes. Her diagnosis in TCM started after the diagnosis of type 1 diabetes, and it was found that the patient had deep deficiency in all the chakras. Treatment was started with Chinese dietary therapy, auricular acupuncture associated with apex ear bloodletting, homeopathy and crystal-based medication.

Results: The three patients presented deficiency of the chakras energy at some point of their lives. The first patient was diagnosed before gestation, the second was diagnosed during gestation and the third was diagnosed after three pregnancies with gestational diabetes and a diagnosis of type 1 diabetes.

Conclusion \& Significance: To prevent and treat GD, it is important to analyze the chakras level of energy and other energy imbalances that are leading to Yin deficiency with Heat retention, the root of the problem, according to TCM. To prevent it is important to treat the patients before the intention of getting pregnant and during the pregnancy, if there are signs of lack of energy, it is important to treat, to prevent the lack of energy on the fetus and other health issues on the mother and the baby.

Keywords: Gestational Diabetes, Energy, Diet, Acupuncture, Traditional Chinese Medicine, Chakras, Homeopathy, Crystal-based medication.

\section{INTRODUCTION}

Gestational diabetes mellitus (GDM) is the most common medical complication of pregnancy. It is associated with maternal and neonatal adverse outcomes. Maintaining adequate blood glucose levels in Gestational Diabetes reduces morbidity for both mother and baby. [1-2]

Around 21.3 million or $16.2 \%$ of live births had some form of hyperglycemia in pregnancy. An estimated $85.1 \%$ were due to gestational diabetes. [3]
The majority of the medical community does not agree on a single set of screening guidelines for gestational diabetes. There are some specialists that would recommend the gestational diabetes only for women older than 25 , or/and with history of diabetes in the family. Others say that screening all pregnant women is the best way to identify all cases of gestational diabetes. [1-2] The screening for gestational diabetes is divided in two parts: initial glucose test and glucose tolerance test. [4-5] 
On the initial test, the patient drinks a syrupy glucose solution. One hour later the patient has a blood test to measure your blood sugar level. A blood sugar level below 130 to 140 milligrams per deciliter $(\mathrm{mg} / \mathrm{dL})$, or 7.2 to 7.8 mill moles per liter $(\mathrm{mmol} / \mathrm{L})$, is usually considered normal on a glucose challenge test, although this may vary by clinic or laboratorial. [4-5]

If blood sugar level is higher than normal, this denotates risk of gestational diabetes and the patient passes through a second exam, the glucose tolerance test. [4-5]

The glucose tolerance test consists in fasting overnight, and then has the blood sugar level measured. After, the patient drinks another sweet solution, with a higher concentration of glucose and the blood sugar level is controlled every hour for three hours. If at least two of the blood sugar readings are higher than normal, the patient is diagnosed with gestational diabetes. [4-5]

In Western Medicine, treatment is usually done with control of the Blood sugar through diet and constant measures. The dietary counselling in Western Medicine consists in eating regularly at starchy and low glycemic index (GI) foods that release sugar slowly - such as whole-wheat pasta, brown rice, granary bread, all-bran cereals, pulses, beans, lentils, muesli and porridge. [4-5]

Is also recommended to eat plenty of fruit and vegetables - aim for at least five portions a day, avoid foods and drinks with sugar. [4-5]

Traditional Chinese Medicine, however, comprehends the gestational diabetes as originated from the same energy imbalances that cause diabetes pathology. The author, in the study entitled why are Diabetic Patients Still Having Hyperglycemia despite Diet Regulation, Antiglycemic Medication and Insulin? Published by the International Journal of Diabetes and Metabolic Disorders, states that for Traditional Chinese Medicine, the energy imbalances associated with the formation of diabetes are deficiency of Yin and Heat retention. This is also the physiopathology for Gestational Diabetes in TCM. [6]

The treatment in Western and Traditional Chinese Medicine has the same goal: to lower the blood glucose. The author used tools from varied ancient medical traditions to create and constitute a treatment that is safe for the pregnant women and the baby, does not contain high-concentrated medications and aims for the overall improvement of the energy state of the patient, before, during and after the gestation. [6]

\section{Purpose}

The purpose of this study is to emphasize the importance of a holistic and energy-based analysis, seeking the importance of chakras and energy imbalances corrections in the prevention and treatment of gestational diabetes.

It is also a goal to present the effectiveness of ancient medical tools in the treatment of the most varied pathologies, in this case, gestational diabetes.

\section{METHODS}

The methodology used was based in three case reports, as well as review of current literature regarding Western and ancient medical tools of diagnosis and treatment of gestational diabetes.

\subsection{Case Report 1}

The first patient, named A.P.S.H, 32-year-old woman, was diagnosed with Gestational Diabetes in the 21 weeks of pregnancy. She was pregnant with twins and was diagnosed after the percentile of the babies was evaluated as too high. The perceptual was above $85 \%$.

After, she performed glucose tolerance test that resulted in 81 in the first hour, 231 in the second hour and $187 \mathrm{mg} / \mathrm{dl}$ in the third hour.

Her chakras were measured a year before the pregnancy, and the resulted in level 1 of 8 , on the energy of all the chakras.

She did treatment for chakras replenishing of energy, for a while before being pregnant, but did not complete the treatment. She performed the treatment for around a month, when she was supposed to perform the treatment for a year or more.

She also presented difficulty in getting pregnant, having to perform IVF for a period of two years, before becoming pregnant. Her husband also had energy deficiency in all the chakras, which was never treated. In another study of the author, entitled Chakra's Energy Deficiency as the Main Cause of Infertility in Women, presented in Osaka, in last June 2019, on the Genecology USA 2019 conference, in Houston, on September 2019. On this study, the author exposes better how many patients with difficulty in getting pregnant have deficiency in the 
chakras energy, and how the replenishing of these energies increased the chances of natural pregnancy, without the need of IVF or other assisted reproduction procedures.

\subsection{Case Report 2}

The second patient, named C., 19-years-old female patient, was diagnosed with gestational diabetes on the 28 week of pregnancy. Her glucose tolerance test appeared $156 \mathrm{mg} / \mathrm{dl}$ (normal being $140 \mathrm{mg} / \mathrm{dl}$ ).

She received chakras measurement during pregnancy, after the diagnoses of Gestational Diabetes, and all her chakras appeared in level 1 of 8 , with severe lack of energy. The patient did not perform the treatment because she had no money to afford the medications.

She had a caesarian session. The baby was supposed to be born of a vaginal birth, but an emergency caesarian session was needed because the umbilical cord was not long enough.

\subsection{Case Report 3}

Patient named C.D.J first got pregnant at age 19. Had a calm pregnancy, able to exercise often and feeling generally well.

On the second pregnancy, the patient reached 110 kilos. She was feeling a lot of swollen and passed through a lot of stress during the beginning of the pregnancy, regarding the health of the baby.

The patient sought an endocrinologist, which diagnosed her with gestation diabetes. The doctor prescribed insulin, but the patient did not intake it immediately. A few days later, she felt strong pain the belly and found out she was bleeding. She was administered buscopan for cramps, the pain did not improve, and moments later, her daughter was born, 37 weeks, weighting 4, 4 kilos. After the birth, the diabetes subsided. In the patient's third pregnancy, she suffered from a lot mental stress, as the pregnancy was accidental. She was oriented by a doctor who once again diagnosed gestational diabetes in the beginning of the pregnancy. She was placed into a weekly pregnancy development program, in which she was accompanied by a doctor and a psychologist.

She was prescribed $30 \mathrm{NPH}$ of insulin in the morning and $15 \mathrm{NPH}$ at night. At thirty-seven week of pregnancy, she was submitted to a caesarian section, due to high glycemia, and lack of fetal movements.
The baby was born with low Apgar, and reanimated a few minutes after birth, when she normalized and cried for the first time.

She was born with 4,2 kilos and low blood sugar. After the pregnancy, the patient was diagnosed with Type 1 Diabetic patient, in 2015.

She started searched for the author's clinic after the diagnosis of type 1 diabetes, in 2015. The patient was using insulin, and had the glycaemia very altered, always around $600 \mathrm{mg} / \mathrm{dl}$, asymptomatic.

She passed through the procedure of chakras measurement, which all appeared in level 1 of 8 . She started treatment with Chinese dietary counselling, auricular acupuncture associated with apex ear bloodletting for rebalancing Yin, Yang, Qi and Blood energies and take off Heat retention. She also started treatment for replenishment the energy of the chakras, with crystal-based medications and homeopathy, according to the Constitutional Homeopathy of the Five Elements according to Traditional Chinese Medicine, developed by the author.

The Chinese dietary counselling she received was to avoid cold beverages, dairy products, raw food, wheat flour, coffee, soda, matte tea. To avoid the formation of internal Heat, it was also recommended for the patient to avoid frying food, milk, coconut, honey, chocolate and alcoholic beverages.

\section{Results}

All the three case reports of the study presented some similarities. The chakras energy deficiencies were constant in all the cases, in different phases of the patients' lives. In the first case, the measurement was done a year before the pregnancy. The second case, she was diagnosed during pregnancy and was not able to perform the treatment, and the third case, the deficiency of the chakras energy was diagnosed after the three pregnancies, after the diagnosis of type 1 diabetes.

In the case of the third patient, the glycaemia diminished from $600 \mathrm{mg} / \mathrm{dl}$ to $150 \mathrm{mg} / \mathrm{dl}$ with the treatment proposed, and the patient had to diminish the dose of insulin by herself to reduce the hypoglycemia crisis. Sometimes the patient has alterations in the glycaemia, going up $200 \mathrm{mg} / \mathrm{dl}$, when she is facing emotional distress.

If not properly treated, the energy deficiency on the chakras center may consolidate as the formation of other chronic diseases, such as 
hypertension, myocardial infarction, allergic reactions, diabetes, etc.

The third patient is still being treated, but feels happier and self-assured, with better results on her work environment, being more patient with the kids she works with, feelings less anxious as well.

\section{DISCUSSION}

Several studies point out the effectiveness of the use of ancient medical tools in the treatment of hyperglycemia. [7-8]

The author, in the already quoted article entitled Why Are Diabetic Patients Still Having Hyperglycemia despite Diet Regulation, Antiglycemic Medication and Insulin?, presents the physiopathology of diabetes in Traditional Chinese Medicine, and promotes the treatment done using Chinese dietary therapy, apex ear bloodletting, auricular acupuncture, homeopathy and crystal-based medications. [6]

The article entitled Why Are Diabetic Patients Still Having Hyperglycemia despite Diet Regulations, Antiglycemic Medication and Insulin?, was published by the author on the International Journal of Diabetes and Metabolic Disorders, in March 2019. [6]

It was also presented in several conferences worldwide. It was presented in Helsinki, Finland, in 2018, on $26^{\text {th }}$ International Diabetes and Healthcare Conference and in Tokyo on the Global Experts Meeting on Infectious Diseases and Diabetes, on February 2019. It was also presented in Vancouver, Canada, on the $6^{\text {th }}$ International Conference on Diabetes Treatment \& Research in October 2019.

The study entitled Syndrome Differentiation of Diabetes by the Traditional Chinese Medicine according to Evidence-Based Medicine and Expert Consensus Opinion, written in 2014 by a group of students of the Guang'anmen Hospital in Beijing, states that "TCM emphasizes individualized treatment and pay attention to Yin-Yang balance and a holistic approach. Deep understanding of diabetes' clinical manifestation and symptom differentiation from different aspects are very important to improve the clinical effects, such as uncomfortable symptoms." [9]
A second study entitled The Therapeutic Effect of Zuogui Wan in Gestational Diabetes Mellitus Ratsattests the effectiveness of the use of the Chinese Herb Zuogui in the treatment of several pregnant women with gestational diabetes. [10]

\subsection{Western Medicine Management and Treatment of Gestational Diabetes}

According to the study entitled Gestational diabetes mellitus written by Eman M. Alfadhli, the physiopathology of gestational diabetes is related to hormones and adipokines, secreted from the placenta, including tumor necrosis factor and human placental growth hormones. Also, according to the author, increased estrogen, progesterone and cortisol during pregnancy is also a factor contributing to high glucose levels. [11]

In the same article, it is stated that the treatment for gestational diabetes include nutritional therapy, exercise and pharmacological interventions such as insulin therapy. The nutrition therapy is based on caloric counts of consumption, and as demonstrated by the patients in the case report, usually is related to consumption of sugar and wheat. [11]

Also, according to the study, exercise appeared to show improvement in the glycemic control. The general recommendation turns around 30 minutes of exercise every day for women with gestational diabetes. [11]

The use of pharmacological interventions is also common, if the medical nutritional therapy and exercise fail. The type and timing of insulin should be chosen based on the specific blood glucose elevation. [11]

The article Gestational diabetes from A to Z, written by Abdel Hameed Mirghani Dirarin 2017, is more focused in the classification of diabetes, and focuses the treatment in new potential insulin mediators. [12]

The study concludes that there are a certain group of women which will have greater risk of presentation gestational diabetes considering weight. However, Abdel attests that the results of his research do not formulate a perfect statement for the association between gestation diabetes and weight. [12]

\subsection{Gestational Diabetes and Ancient Medical Tools}

In Traditional Chinese Medicine, Gestational Diabetes has the same physiopathology that 
diabetes itself: Yin deficiency and Heat Retention. In the three case reports proposed, the patients had deficiency in the chakra's energy, in the same way as other diabetic patients, described by the author in her article entitled Why Are Diabetic Patients Still Having Hyperglycemia despite Diet Regulation, Antiglycemic Medication and Insulin?, published by the International Journal of Diabetes and Metabolic Disorders, in March 2019.Therefore, the hypothesis here proposed is that the chakra's energy deficiency may be one of the several underlying causes of gestational diabetes, as it is the underlying cause of diabetes type 1 or 2. [6]

The first two patients were not able to perform the treatment to replenish the energy of the chakras. However, as they both had chakras energy deficiency and gestational diabetes, this opened a possibility of inquiring if the two things could be associated, and could chakras energy deficiency be linked to the formation of gestational diabetes.

The propositions of treatment and diagnosed were based in ancient medical tools literature, such as Hippocratic Medicine, Traditional Chinese Medicine and Ayurvedic Medicine to propose a treatment that is holistic and energybased. [6]

To better understand the treatment proposed it is important to firstly stablish the principles of Traditional Chinese Medicine.

\subsubsection{Cornerstones of Traditional Chinese Medicine: Yin and Yang and Five Elements Theory}

Other articles of the author, as well as the books Essentials of Traditional Medicine, I-Ching or the Yellow Emperor demonstrate the theory of Yinand Yang and its relationship with the internal energies of the body. [13-15]

On the theory, Yin and Yang energies are present in everything in the universe. The idea of the symbol presented in Figure 1, as well as the theories behind is to state the opposite complementary statement. Examples would be day and night, male and female, and more specifically, in the human body. [13-15]

A balanced life style would promote health, but this balance would be based on the internal energies state. According to TCM, the human body has Yin and Yang energies, primordial to the functioning of the body, and distributed through several meridians throughout the body. [13-15]

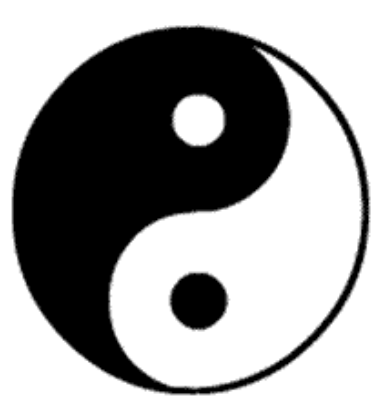

Figure1. Yin and Yang

To explain this distribution, Traditional Chinese Medicine uses the Five Elements Theory. The Five Elements Theory, in the same way as the Yin and Yang theory, its anchored in the idea of cycles and balance between elements. In this case, the elements considered are Wood, Fire, Earth, Metal and Water, as proposed in Figure 2. [13-15]

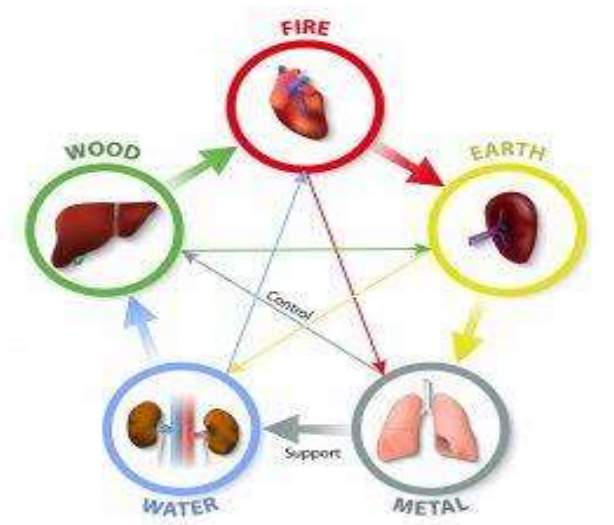

Figure2. Five Elements Theory

Each of these elements is related to one specific organ of the body. These organs are called massive organs and they are believed to be center of flow and functioning of energy of the body. Each of these organs has also a specific balance related to Yin and Yang. [13-15]

Each element is linked to a massive organ, a hollow organ, a surface part, an opening, a trait, a mental activity and a taste. In this way, TCM proposes a way of looking to the functioning of the human body as always systemic, an imbalance in the Yin energy of the body, will reflect in the whole body, through the symptom proposed. [13-15]

Despite the Yin and Yang energy, there are more two energies flowing through the organs of the Five Elements Theory and through all the 12 meridians of energy in the body. These energies are $Q i$ and Blood. The $Q i$ energy was conceptualized in other ancient medical cultures 
as well, including Ayuervedic Medicine. This energy, also known as prana, or vital energy is also flowing throughout the body, and its flow is equally important. [16] The last energy is the Blood energy, responsible for the functioning of Blood and the distribution of energy on the body. [13-15] Therefore, the balance considered by Traditional Chinese Medicine that would promote health, is based on the functioning of four energies together Yin, Yang, $Q i$ and Blood energies, as showed in Figure 3. These energies have to be balanced, and flowing on the Five Elements present inside the body, to promote health. [17-22]

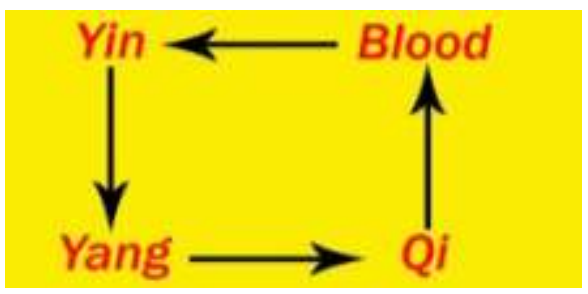

Figure3. Yin, Yang, Qi and Blood Relationship

Symptoms, as previously said, are imbalances in these internal energies. When one energy of the four ones in Figure 3 is imbalanced, it wills imbalance the other in a dynamic cycle. The use of ancient medical tools such as auricular acupuncture, apex ear bloodletting has the goal of rebalancing the energies of the body.

\subsubsection{The root-level treatment for gestational diabetes}

The treatment of the patients in the case reports, as well as other patients of the author, was based on the analysis of one specific case the author had in 2007. The patient in question presented symptoms of pain in the legs, and received treatment with auricular acupuncture and systemic acupuncture associated with apex ear bloodletting and Chinese Dietary counselling to treat these symptoms, according to his energy disturbances. In the specific case of this patient, the energy disturbance was Kidney Yang deficiency. [21-22]

The patient then presented improvement of his pain in the legs after 10 auricular acupuncture sessions, and returned to the clinic to be evaluated. After the interview, he reveled to the author that he also had major improvement in a symptom the author was not aware he had: he was being treated for Glaucoma in the last 40 years. The treatment for Kidney Yang deficiency not only improved his symptoms of pain in the legs, but diminished his intraocular pressure, from $40 \mathrm{mmHg}$ to $17 \mathrm{mmHg}$. [21-22]

To explain the differences between Western and Traditional Chinese Medicine, the author created the metaphor of the tree. In this tree, the tree has a trunk with several branches. Each branch represents one medical specialty and each leaf coming out of each branch represent the symptoms and diseases of each specialty. The leaves and the branches are the visible part and the object of study and practice in the western medical profession. The focus is to treat each leaf in each specialty. The treatment proposed aims to treat the entire tree, through the root, that is invisible to the naked eye, responsible for gestational diabetes. [21-22]

There, in this root, there is energy that when balanced can generate health to the whole tree. The reason of this study is to address which are the energy alterations present in the root level, responsible for gestational diabetes. [21-22]

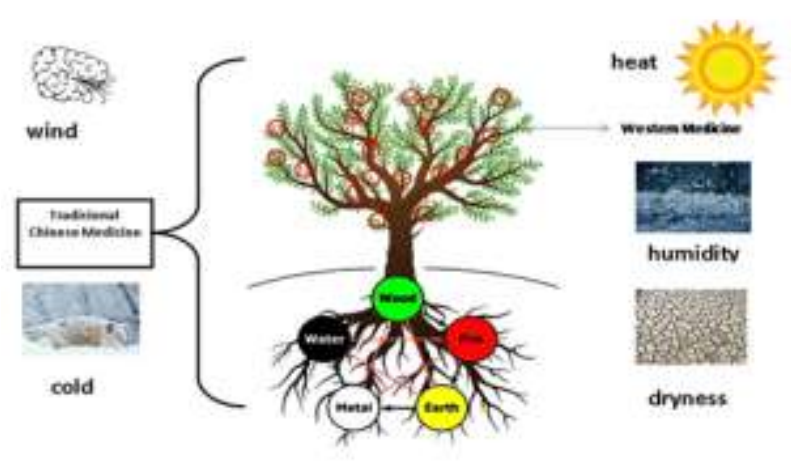

Figure4. Schematic Drawing of the Views of Western and Traditional Chinese Medicine

The main proposal of the author is to approach the diseases from the energy imbalances first, considering the root first, or seeing the tree upside down.
The three case reports reinforce the importance of observing the patient holistically and treating the symptoms in their root-level. 


\subsection{Five Elements Theory and Chakras Theory: Where ancient medical theories overlap}

The chakras concept, also already presented by the author in several other articles, appears in the Aryuveda medical theories, as well as in Yoga theories. Chakras are main points of energy concentration. There are seven main chakras present in the body and their relationship among themselves is extremely important for the body's health. [23]

The chakras correspond to the Five Elements within the Five Elements Theory, as exposed in small trials in literature. Many ancient medical scholars believe that the five elements theory and the chakras theory can connect. One example is entitled The Geometry of Emotions: Using Chakra Acupuncture and 5-Phase Theory to Describe Personality Archetypes for Clinical Use, the author links the use of chakras

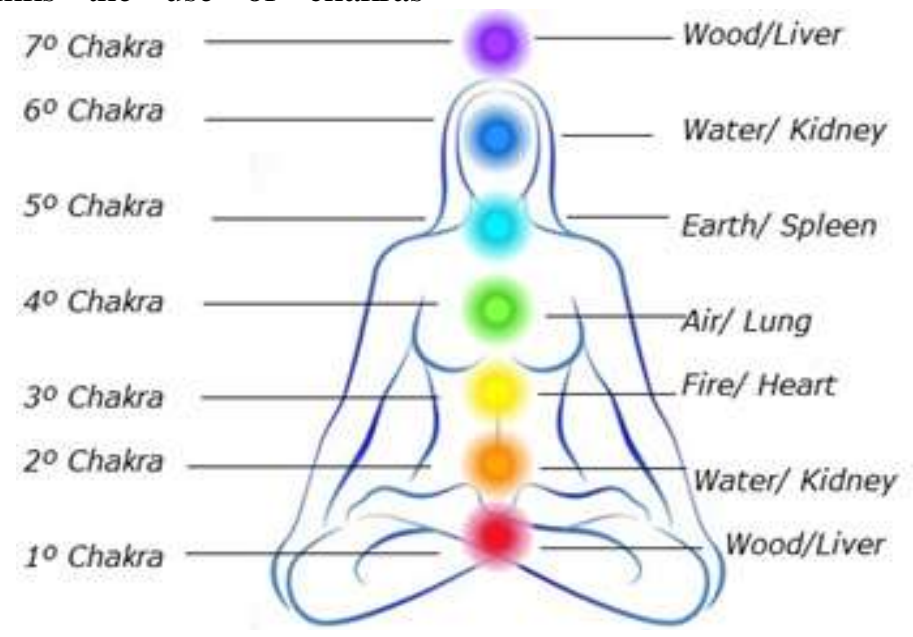

Figure5. Chakras Energy Correspondence

The use of the chakra centers measurement was previously analyzed by George Washington, in the article entitled The Scientific Basis of Integrative Medicine. In the article, the author describes the pineal gland as a link between our external environment and the network of internal body systems, comprehending the chakras system as an extension to the body's working systems. [24] What the three cases presented on this study had in common was the deficiency of all the chakras energy, with exception of the seventh. Despite the patients not have received treatment for this energy deficiency during gestation, the author had the goal of showing that this chakra's energy deficiency is a common aspect in patients with gestational diabetes, and is also a common factor on not pregnant patients with diabetes. This energy alteration can be measured through measurement with the principles of Traditional Chinese Medicine, described by the author in other articles, to comprehend the influence of a weakened result in the chakras in the other systems of the body. Although this article is focused in personality archetypes linked to the chakras, it establishes a relationship between the Five Elements Theory and the seven chakras, in the same way as proposed by the author in previous articles. [23]

As there are seven chakras and five elements, the seventh chakra is ruled by the first (Wood or Liver), and the sixth chakra is ruled by the second (Water or Kidney). The fifth chakra is ruled by Earth (Spleen-Pancreas), the fourth is ruled by Air, or Lung, the third is ruled by Fire or Heart. The second is ruled by Water or Kidney and the first chakra is ruled by Wood or Liver, as shown in Figure 5. [21-22] the procedure of radiesthesia and is very probable that this might be the beginning of the imbalances that may lead to the formation of other diseases, in this case diabetes (gestational or type 1 or 2 ).

Therefore, it is possible to propose that there is a relationship between chakras energy imbalances and the formation of gestational diabetes.

\subsection{Arndt-Schultz Law}

In this article, what the author aims to demonstrate is that patients with gestational diabetes have deep energy deficiencies leading to the formation of internal Heat, generating alterations on the glycaemia during the gestation period. The use of medications in highconcentrations during pregnancy, may harm even deeper the vital energy, because according 
to the Arndt-Schultz's law, all drugs in high concentrations cause a reduction of vital energy, as showed in Figure 6. This reduction would generate the patient's energy weakness and the formation of internal Heat due to energy imbalances. [17-22]

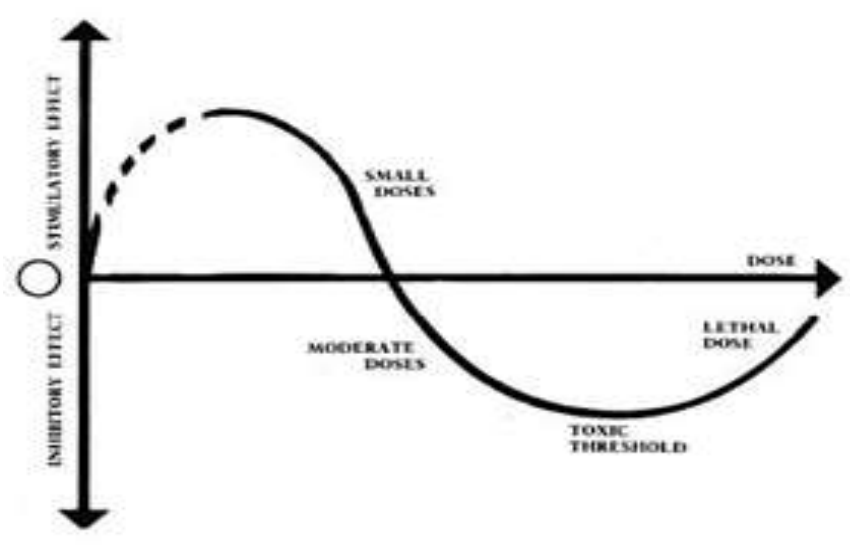

Figure6. Arndt-Schultz Law

Because of this, the author prefers high-diluted medications (homeopathy) in order to promote improvement on the vital energy, reducing the chances of weakening the health of the mother and baby and the formation of other energy imbalances, which may lead to other diseases such as gestational diabetes. Homeopathy is considered a medical specialty in Brazil since 1985.

\section{Conclusion}

The conclusion of this study is that when we are faced with a pregnant woman, who has alterations in the glycaemia; the glycemia increase is only a symptom of an energy imbalance, suffered by the patient. This is why the study of this patient in the energy level is important to treat the root of the problem, and not only the symptom, that is the hyperglycemia. If the pregnant woman has little energy in the chakras, this can lead to an energy alteration in the fetus formation, the baby may be born with an energy deficiency, acquired from the mother and from the father.

The ideal would be to treat in the energy level, all women before pregnancy, so the prevention of gestational diabetes could be done in the rootlevel. Treating the couple before conception is also significant to the energy level of the fetus. If both parents have weak energy of the chakras, the baby could already be born with energy imbalances, generated due to the energy deficiencies passed by both parents.

\section{REFERENCES}

[1] Hanne Melchior. The Prevalence of Gestational Diabetes: A Population-Based Analysis of a Nationwide Screening Program. DtschArztebl
Int. 2017 Jul; 114(24): 412-418.Published online 2017 Jul 16. doi: 10.3238/arztebl.2017.0 412

[2] Kai Wei Lee, SiewMooiChing, Vasudevan Ramachandran, Anne Yee, Fan KeeHoo,Yook Chin Chia, Wan Aliaa WanSulaiman, SubapriyaSuppiah, MohdHazmi Mohamed, and Sajesh K. Veettil. Prevalence and risk factors of gestational diabetes mellitus in Asia: a systematic review and meta-analysis,. BMC Pregnancy Childbirth. 2018; 18: 494. Published online 2018 Dec 14. doi: 10.1186/s12884-018-2131-4

[3] International Diabetes Federation. Care \& Prevention - Gestational Diabetes.

[4] Rani PR1, Begum J. Screening and Diagnosis of Gestational Diabetes Mellitus, Where Do We Stand. J ClinDiagn Res. 2016 Apr;10(4):QE014. doi: 10.7860/JCDR/2016/17588.7689. Epub 2016 Apr 1.

[5] Aktün HL1, Uyan D1, Yorgunlar B1, Acet M. Gestational diabetes mellitus screening and outcomes. J Turk GerGynecol Assoc. 2015 Mar 1; 16(1):25-9. doi: 10.5152/jtgga.2015.15081. eCollection 2015.

[6] Huang Wei Ling. Why Are Diabetic Patients Still Having Hyperglycemia despite Diet Regulation, Antiglycemic Medication and Insulin? International Journal of Diabetes \& Metabolic Disorders.

[7] Maggie B. Covington. Traditional Chinese Medicine in the Treatment of Diabetes. Diabetes Spectrum 2001 Aug; 14(3): 154-159. https://doi.org/10.2337/diaspect.14.3.154

[8] Zhijun Wang. Treating Type 2 Diabetes Mellitus with Traditional Chinese and Indian Medicinal Herbs

[9] Jing Guo, Hongdong Chen, Jun Song, Jia Wang, Linhua Zhao. Syndrome Differentiation of Diabetes by the Traditional Chinese Medicine according to Evidence-Based Medicine and Expert Consensus Opinion. Evidence-Based 
The importance of Chakras and Energy Imbalances Correction in the Prevention and Treatment of Gestational Diabetes

Complementary and Alternative Medicine Volume 2014, Article ID 492193, 7 pages http://dx.doi.org/10.1155/20 14/492193

[10] Yuwei Wang, Qianjin Feng, Xin Niu, Xinshe Liu, Kaixia Xu, Xiangzhu Yang, Huifeng Wang, and Qiuju Li. The Therapeutic Effect of Zuogui Wan in Gestational Diabetes Mellitus Rats. J Anal Methods Chem. 2014; 2014: 737961. Published online 2014 Jul 22. doi: 10.1155/2014/737961

[11] Eman M. Alfadhli. Gestational diabetes mellitus. Saudi Med J. 2015; 36(4): 399-406. doi: 10.15537/smj.2015.4.10307

[12] Abdel Hameed Mirghani Dirar. Gestational diabetes from A to Z. World J Diabetes. 2017 Dec 15; 8(12): 489-511. Published online 2017 Dec 15. doi: 10.4239/wjd.v8.i12.489

[13] OuYang B, Gu Z (1996) Essentials of Traditional Chinese Medicine. Shandong, Science and Technology Press.

[14] HUA-CHING-NI. I CHING: The book of Changes and the Unchanging Truth. 2004.

[15] Maoshing NI, The Yellow Emperor's Classic of Medicine: The Essential Text of Chinese Health and Healing. Boston, Massachusetts, 2015. Shambala.

[16] James Flowers. What Is Qi? Evid Based Complement Alternat Med. 2006 Dec; 3(4): 551-552. Published online 2006 Oct 23. doi: 10.1093/ecam/nel074
[17] Huang Wei Ling. "Can Recurrent Furunculosis is treated without the Use of Antibiotics?"Acta Scientific Microbiology Volume 1, Issue 9 (2018): 04-12.

[18] Huang Wei Ling. "Could Postsurgical Nosocomial Cellulitis is treated without the Use of Antibiotics?"Acta Scientific Microbiology 1.9 (2018):

[19] Huang W.L. Can Hospital Osteomyelitis Be Treated Without the Use of Antibiotics? Into J Microbiol Infect Dis. 2018; 2(1):1-6.

[20] Huang WL (2018) The Treatment of Asthma Based on Traditional Chinese Medicine and Homeopathy. J Pediat Infants. Vol: 1, Issu: 1 (23-27).

[21] Huang WL (2019) Is It Possible to Treat Giant Congenital Hairy Melanocytic Nevus Clinically? J Clin Case Rep Trials. Vol: 2, Issu: 2 (06-13).

[22] Ling HW (2019) Can We Treat Atopic Dermatitis without using Corticosteroids? J Pediat Infants Vol: 2, Issu: 1 (08-19).

[23] Chase CR. The Geometry of Emotions: Using Chakra Acupuncture and 5-Phase Theory to Describe Personality Archetypes for Clinical Use. Med Acupuncture. 2018 Aug 1; 30(4):167-178. doi: 10.1089/acu. 2018.1288.

[24] George Washington. The basis of Integrative Medicine.

Citation: Huang Wei Ling. The importance of Chakras and Energy Imbalances Correction in the Prevention and Treatment of Gestational Diabetes. ARC Journal of Diabetes and Endocrinology. 2019; 5(2):12-20. doi:dx.doi.org/10.20431/2455-5983. 0502003.

Copyright: (c) 2019 Authors. This is an open-access article distributed under the terms of the Creative Commons Attribution License, which permits unrestricted use, distribution, and reproduction in any medium, provided the original author and source are credited. 